\title{
Statistical analysis of inter-cell interference in uplink OFDMA networks with soft frequency reuse
}

Yuanping Zhu ${ }^{1,3}$, Jing $\mathrm{Xu}^{1,2^{*}}$, Jiang Wang ${ }^{1,2}$ and Yang Yang ${ }^{1,2}$

\begin{abstract}
As the inter-cell interference becomes a great challenge in frequency reuse one systems, soft frequency reuse (SFR) has been widely used to deal with the severe inter-cell interference especially for cell edge users. This paper proposes an analytical method to investigate the statistics of inter-cell interference in uplink orthogonal frequency division multiple access systems when SFR scheme is adopted. Probability density functions of inter-cell interference in different frequency bands are derived and then used to deduce the expectation and variance of inter-cell interference from multiple interfering cells. The derivations are validated through numerical results. In addition, the relationship between system parameters and the statistics of inter-cell interference in different frequency bands is investigated. These contributions will give insights and guidelines for the system optimization.
\end{abstract}

\section{Introduction}

Orthogonal frequency division multiple access (OFDMA) has been adopted in many wireless networks such as IEEE 802.16 and the 3rd Generation Partnership Project long-term evolution (LTE), due to many beneficial characteristics. Meanwhile, as the limited frequency resource becomes a bottleneck for the increasing data rate demand, it would be better to reuse the available frequency among each cell. However, inter-cell interference (ICI) will be more severe for user equipments (UEs) in the cell edge region. Many inter-cell interference coordination (ICIC) techniques have been proposed to mitigate the problem. One typical solution introduced in LTE [1] is soft frequency reuse (SFR): (1) UEs in the cell center region which experience/generate low interference and require low power to communicate with their serving evolved NodeBs (eNBs) are permitted to use the whole spectrum, and (2) UEs in the cell edge region which experience/generate strong interference and require high power to ensure reliable communication are constrained to be scheduled with

\footnotetext{
*Correspondence: jing.xu@shrcwc.org

1 Key Laboratory of Wireless Sensor Network \& Communication, Shanghai Institute of Microsystem Information and Technology (SIMIT), Chinese

Academy of Sciences (CAS), Shanghai 200050, People's Republic of China

2 Shanghai Research Center for Wireless Communications (WiCO), Shanghai

200335, People's Republic of China

Full list of author information is available at the end of the article
}

a part of the spectrum, while these resources should be allocated to UEs in the center region or not be used in neighboring cells. With such resource scheduling limitation among adjacent cells, SFR can be utilized to avoid severe ICI. Figure 1 demonstrates an example of the SFR scheme for multicell cellular networks.

If statistical characteristics of ICI can be derived through theoretical analysis, time-consuming systemlevel simulations may be avoided. Therefore, researches about statistics of ICI have received more and more consideration recently. Some of them focus on analyzing the statistics of signal-to-interference ratio or signalto-interference-and-noise ratio (SINR) analytically [2-6]. Others concern the probability density function (PDF) of ICI or SINR. The conditional PDF of carrier-based interference plus noise in downlink OFDMA networks is derived in [7]. In [8,9], the PDFs of SINR and interference are derived through analyzing the samples of systemlevel simulations and checking several given hypotheses, without deriving and validating the corresponding closedform expressions. In [10], the PDF of downlink SINR in randomly located femtocells is given by analysis and calculation. Recently, many analytical frameworks are presented to evaluate the distribution of downlink SINR based on the Poisson point process modeling of nodes $[11,12]$. These papers focus on the statistical analysis of the downlink ICI in cellular networks.

\section{Springer}

(c) 2013 Zhu et al: licensee Springer. This is an Open Access article distributed under the terms of the Creative Commons Attribution License (http://creativecommons.org/licenses/by/2.0), which permits unrestricted use, distribution, and reproduction in any medium, provided the original work is properly cited. 


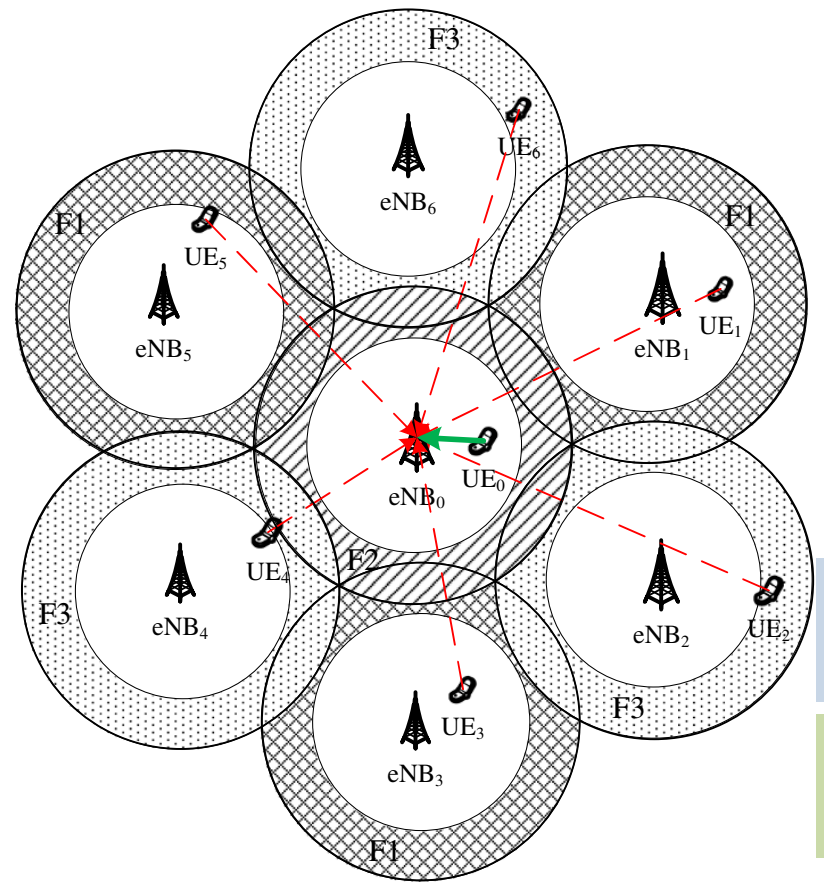

$\stackrel{\text { Interference }}{\rightarrow}$

$\underset{\text { Uplink signal }}{\longrightarrow}$

Part Frequency for

cell edge users

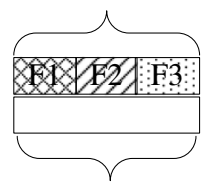

Full frequency for

cell centre users

For sub-band $\mathrm{F} 1$

Set-A: $\left\{\mathrm{eNB}_{2}, \mathrm{eNB}_{4}, \mathrm{eNB}_{6}\right\}$

Set-B: $\left\{\mathrm{eNB}_{1}, \mathrm{eNB}_{3}, \mathrm{eNB}_{5}\right\}$

For sub-band F3

Set-A: $\left\{\mathrm{eNB}_{1}, \mathrm{eNB}_{3}, \mathrm{eNB}_{5}\right\}$

Set-B: $\left\{\mathrm{eNB}_{2}, \mathrm{eNB}_{4}, \mathrm{eNB}_{6}\right\}$

Figure 1 Uplink interference model when SFR is adopted in cellular networks.

It is worth noting that in the downlink, the sources of interference are eNBs which are fixed in cellular networks, thus the scheduling scheme almost has no impact on the ICI once the frequency reuse scheme is determined. However, the uplink interfering sources are UEs which may be located anywhere in a cell. Moreover, in different scheduling periods, an uplink frequency band may be allocated to different UEs. As a result, the uplink interference will show great fluctuation, and the analysis of the downlink interference cannot be applied to analyze the uplink interference directly. In [13], the PDF of SINR in ad hoc networks is derived. Only one interferer is considered in this scenario, and the derived results may not be applied to cellular networks. The uplink ICI is investigated in [14] by generating amounts of samples and then drawing a histogram without deriving a closed-form expression. The uplink coverage probability in cellular networks is derived in [15] when the power control scheme is involved, and all the nodes are assumed to be randomly placed.

In this paper, the fixed infrastructure and randomly distributed UEs are considered in the model of cellular networks with SFR scheme. And for the channel model, the path loss, shadowing, and Rayleigh fading are included. Based on such system assumptions, the PDFs of ICI in different frequency bands are derived and used to deduce the closed-form expressions for the expectation and variance. Furthermore, the impacts of many system parameters on the PDF, expectation, and variance of ICI are investigated.
The rest of the paper is organized as follows. Section 2 introduces the system model. The PDF, expectation, and variance of the uplink ICI in the frequency band allocated to cell edge UEs in the cell of interest are derived in section 3. And the corresponding statistical analysis of the uplink ICI in the rest frequency bands are given in section 4 . In section 5 , the numerical results are demonstrated, and the influences of system parameters on the uplink ICI are studied. Finally, conclusions are made in section 6 .

\section{System model}

Considering that in the actual deployment of cellular networks, the infrastructures are always fixed while UEs are randomly distributed, and the propagation attenuation is related to the distance. It is rational to model the coverage area of a cell as a circular region. For simplicity, the model of cellular network consists of a cell of interest and its neighboring cells as depicted in Figure 1; the serving eNB in the cell of interest is defined as $\mathrm{eNB}_{0}$, and the other eNBs are denoted as $\operatorname{eNB}_{i}(i=1,2, \ldots, 6)$. Correspondingly, in each cell, the UE which is scheduled with a given frequency band is denoted as $\mathrm{UE}_{i}$. Each cell is divided into two parts: the inner part which represents the cell center region, and the outer part which represents the cell edge region. Accordingly, the UEs in the cell edge region are defined as exterior UEs, and the UEs in the cell center region are defined as interior UEs. 
For the SFR scheme, the whole frequency band is divided into three sub-bands: F1, F2, and F3 as shown in Figure 1. Exterior UEs are constrained to use one of the sub-bands with high transmit power $P_{\mathrm{t}, 1}$, while the interior UEs can reuse the whole frequency band with a reduced power $P_{\mathrm{t}, 0}\left(P_{\mathrm{t}, 0}<P_{\mathrm{t}, 1}\right)$. In this paper, $P_{\mathrm{t}, 1}$ is set as the full transmit power. UEs are assumed to be uniformly distributed in the cellular networks, and all the frequency bands are allocated to active UEs in every cell. In addition, eNB schedule frequency resources independently under the premise of allocating orthogonal sub-bands to cell edge UEs among adjacent cells.

In uplink, $\mathrm{eNB}_{0}$ receives the desired signal from $\mathrm{UE}_{0}$ and interference from UEs in the neighboring cells. The signal link is represented by green solid arrow while the interference links are represented by red dashed arrows in Figure 1.

For the channel model, the distance-dependent path loss, shadowing, and Rayleigh fading are considered. These three parts are independent. The shadowing is modeled as a lognormal random variable (RV), and the gain related to Rayleigh fading is modeled as an exponential-distributed RV. Besides, we assume that the antenna pattern of both UEs and eNBs are omnidirectional. Therefore, the uplink interference power from $\mathrm{UE}_{i}$ can be described as

$$
I_{i}=P_{\mathrm{t}} \alpha D_{i}^{-\beta} e^{\lambda \xi} H
$$

where $I_{i}$ indicates the received interference at $\mathrm{eNB}_{0}, P_{\mathrm{t}}$ denotes the transmit power of $\mathrm{UE}_{i}$ which equals to either $P_{\mathrm{t}, 0}$ or $P_{\mathrm{t}, 1}, D_{i}$ represents the distance between $\mathrm{eNB}_{0}$ and $\mathrm{UE}_{i}, \alpha$ and $\beta$ are the path loss constant and exponent, respectively, $\alpha D_{i}^{-\beta}$ represents the path loss, $\xi$ represents the logarithmic shadowing in the unit of $\mathrm{dB}$ which is assumed to be a zero-mean Gaussian RV with variance $\sigma^{2}$, $\lambda=\ln (10) / 10$, and $H$ represents the gain related to the Rayleigh fading.

As the SFR scheme is adopted, the distribution of interference in sub-bands F2 and F1/F3 (we define F1/F3 which represents 'F1 or F3' for convenience) will be different. In the following, they will be analyzed separately.

\section{Statistical analysis of the uplink $\mathrm{ICl}$ in sub-band F2}

Since the neighboring cells have identical relative locations to the cell of interest, it would be tractable to analyze the interference from one interfering cell at first and then extend to the multiple interfering cells' scenario.

As shown in Figure 2, the cell radius is $R$, and the intersite distance is $\sqrt{3} R$; $\mathrm{eNB}_{0}$ is located at the original point $(0,0)$, and $\mathrm{eNB}_{i}$ is located at $(\sqrt{3} R, 0)$. In the interfering cell, the inner disk represents the cell center region, the green annular area represents the cell edge region, and $R_{1}=\gamma R(0 \leq \gamma \leq 1)$ indicates the radius of the cell center region. The overlapping area between the cell center region of the interfering cell and the circular region $x^{2}+y^{2} \leq D_{i}^{2}$ is defined as $\Delta_{1}$, while $\Delta_{2}$ denotes the overlapping area between the cell edge region of the interfering cell and the circular region $x^{2}+y^{2} \leq$ $D_{i}^{2}$.

Define $X=e^{\lambda \xi}, Y=\alpha D_{i}^{-\beta}$, and suppose that the transmit power of UE is given, the random part of $I_{i}$ is $X Y H$,

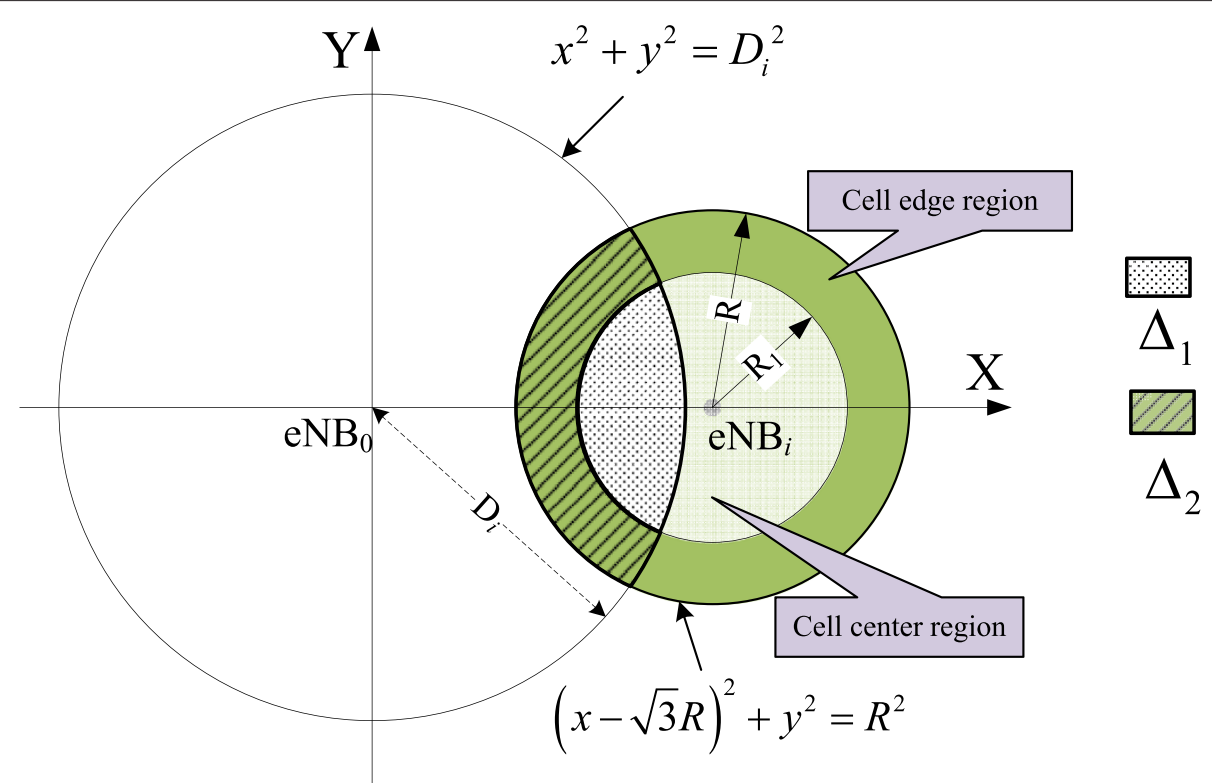

Figure 2 Single adjacent interfering cell model. 
where $X, Y$, and $H$ are independent. The PDF of the product of two independent RVs (e.g., $Z=X Y$ ) is given by

$$
f_{Z}=\int_{X} \frac{1}{x} f_{X}(x) f_{Y}(z / x) d x
$$

This could be used to derive the PDF of $I_{i}$ if the PDFs of $X$, $Y$, and $H$ are obtained.

The distribution of the shadowing $\xi$ is modeled as zeromean Gaussian distribution which is denoted as $N\left(0, \sigma^{2}\right)$, thus, the PDF of $X=e^{\lambda \xi}$ can be derived as

$$
f_{X}(x)=\frac{1}{\sqrt{2 \pi} \sigma \lambda x} \exp \left[-\frac{(\ln x)^{2}}{2 \lambda^{2} \sigma^{2}}\right], x>0
$$

Since $H$ represents the power gain of Rayleigh fading, the PDF of $H$ is modeled as

$$
f_{H}(h)=\mu e^{-\mu h}, h>0
$$

where $\mu$ is the rate parameter and is always set as 1 traditionally.

\subsection{PDF of the $\mathrm{ICl}$ in sub-band $\mathrm{F} 2$}

For convenience, we define $\mathrm{L}_{\text {center }}$ represents the event that $\mathrm{UE}_{i}$ locates in the cell center region and $\mathrm{L}_{\text {edge }}$ represents the event that $\mathrm{UE}_{i}$ locates in the cell edge region. From Figure 1, it is obvious that the received interference at $\mathrm{eNB}_{0}$ in sub-band $\mathrm{F} 2$ comes from the interior UEs of the neighboring cells, which means that the event $\mathrm{L}_{\text {center }}$ occurs. Therefore, from Figure 2, the conditional cumulative distribution function (CDF) of $D_{i}$ equals to the proportion of the area of $\Delta_{1}$ to the area of cell center region. That is

$$
F_{d}\left(D_{i} \mid \mathrm{L}_{\text {center }}\right)=\frac{S_{\Delta_{1}}}{\pi \gamma^{2} R^{2}}
$$

$S_{\Delta_{1}}$ represents the area of $\Delta_{1}$ in Figure 2 and is shown as

$$
\begin{aligned}
S_{\Delta_{1}}= & D_{i}^{2} \arccos \left(\frac{D_{i}^{2}+\left(3-\gamma^{2}\right) R^{2}}{2 \sqrt{3} D_{i} R}\right) \\
& +\gamma^{2} R^{2} \arccos \left(\frac{\left(3+\gamma^{2}\right) R^{2}-D_{i}^{2}}{2 \sqrt{3} \gamma R^{2}}\right) \\
& -\frac{\sqrt{\left(6+2 \gamma^{2}\right) R^{2} D_{i}^{2}-D_{i}^{4}-\left(3-\gamma^{2}\right)^{2} R^{4}}}{2}
\end{aligned}
$$

where $(\sqrt{3}-\gamma) R \leq D_{i} \leq(\sqrt{3}+\gamma) R$. Furthermore, the conditional PDF of $\mathrm{D}_{i}$ equals to the first derivative of CDF, and it is given by

$$
f_{d}\left(D_{i} \mid \mathrm{L}_{\text {center }}\right)=\frac{2 D_{i}}{\pi R^{2} \gamma^{2}} \arccos \left(\frac{D_{i}^{2}+\left(3-\gamma^{2}\right) R^{2}}{2 \sqrt{3} R D_{i}}\right)
$$

Based on (7), it is easy to derive the conditional PDF of the path loss $Y=\alpha D_{i}^{-\beta}$ as

$$
\begin{aligned}
f_{Y}\left(y \mid \mathrm{L}_{\text {center }}\right)= & \frac{2 y^{-2 / \beta-1} \alpha^{2 / \beta}}{\pi R^{2} \gamma^{2} \beta} \\
& \times \arccos \left[\frac{\alpha^{1 / \beta} y^{-1 / \beta}}{2 \sqrt{3} R}+\frac{\left(3-\gamma^{2}\right) R y^{1 / \beta}}{2 \sqrt{3} \alpha^{1 / \beta}}\right]
\end{aligned}
$$

where $\alpha[(\sqrt{3}+\gamma) R]^{-\beta}<Y<\alpha[(\sqrt{3}-\gamma) R]^{-\beta}$. Define $E_{1}=\frac{2 \alpha^{2 / \beta}}{\pi R^{2} \gamma^{2} \beta}, E_{2}=\frac{\alpha^{1 / \beta}}{2 \sqrt{3} R}, E_{3}=\frac{\left(3-\gamma^{2}\right) R}{2 \sqrt{3} \alpha)^{1 / \beta}}$, the conditional PDF of $Y$ is

$f_{Y}\left(y \mid \mathrm{L}_{\text {center }}\right)=E_{1} y^{-2 / \beta-1} \times \arccos \left[E_{2} y^{-1 / \beta}+E_{3} y^{1 / \beta}\right]$

The interference from the interior UEs in a neighboring cell is $I_{i}=P_{\mathrm{t}, 0} X Y H$, and its PDF is derived as

$$
\begin{aligned}
f_{I_{i}}\left(I \mid \mathrm{L}_{\text {center }}\right)= & \frac{1}{P_{t, 0}} \int_{0}^{\infty} \frac{1}{h} f_{H}(h) \int_{0}^{\infty} \\
& \frac{1}{x} f_{X}(x) f_{Y}\left[I /\left(P_{t, 0} x h\right) \mid \mathrm{L}_{\text {center }}\right] d x d h
\end{aligned}
$$

Suppose that $N$ represents the number of neighboring cells, $I_{F 2}=\sum_{i=1}^{N} I_{i}$ is the total received interference in sub-band F2 from multiple neighboring cells. As mentioned in section 2, each cell schedule frequency resources independently. This indicates that in sub-band F2, the ICI from the different neighboring cells are independent and identically distributed (i.i.d.) random variables. With the PDF of the ICI from single neighboring cell, the PDF of $I_{\mathrm{F} 2}$ is calculated by the $N$ th order convolution of (10) as shown in (11), where " " represents the convolution. The convolution can be calculated using numerical calculation methods.

$$
f_{I_{\mathrm{F} 2}}=\underbrace{f_{I_{i}}\left(I \mid \mathrm{L}_{\text {center }}\right) * \cdots * f_{I_{i}}\left(I \mid \mathrm{L}_{\text {center }}\right)}_{N-\text { th order convolution }}
$$

\subsection{Expectation and variance of the uplink $\mathrm{ICl}$ in sub-band F2}

As we know, many statistics of random variables will demonstrate some important properties. For example, the expectation of ICI can reflect the mean interference level, and the variance of ICI will give insight into the fluctuation of interference. In the following context, the closed-form expressions of expectation and variance of ICI in sub-band F2 will be derived respectively. 
Since the shadowing $X$, path loss $Y$, and Rayleigh fading $H$ are independent, the $m$ th moment of $I_{i}$ could be calculated as

$$
E\left[\left(I_{i}\right)^{m}\right]=E\left[\left(P_{\mathrm{t}} X Y H\right)^{m}\right]=\left(P_{\mathrm{t}}\right)^{m} E\left[X^{m}\right] E\left[Y^{m}\right] E\left[H^{m}\right]
$$

where $E\left[()^{m}\right]$ represents the $m$ th moment of the corresponding RV.

To get closed-form expressions for the moments of $Y$, we use the power series of arccosine function to derive an instituted expression of $f_{Y}\left(y \mid \mathrm{L}_{\text {center }}\right)$.The instituted expression is

$$
\begin{aligned}
f_{Y}\left(y \mid \mathrm{L}_{\text {center }}\right)= & E_{1} y^{-2 / \beta-1} \arccos \left[E_{2} y^{-1 / \beta}+E_{3} y^{1 / \beta}\right] \\
= & E_{1} y^{-2 / \beta-1}\left\{\frac{\pi}{2}-\sum_{n=0}^{\infty} G_{n}\left(E_{2} y^{-1 / \beta}\right.\right. \\
& \left.\left.+E_{3} y^{1 / \beta}\right)^{2 n+1}\right\} \\
= & E_{1} y^{-2 / \beta-1}\left[\frac{\pi}{2}-\sum_{n=0}^{\infty} G_{n} \sum_{p=0}^{2 n+1} \frac{(2 n+1) !}{p !(2 n+1-p) !}\right. \\
& \left.\times\left(E_{2} y^{-1 / \beta}\right)^{p}\left(E_{3} y^{1 / \beta}\right)^{2 n+1-p}\right] \\
= & \left.\frac{\pi}{2} E_{1} y^{-2 / \beta-1}\right] \\
& -E_{1} \sum_{n=0}^{\infty} G_{n} E_{3}^{2 n+1} \sum_{p=0}^{2 n+1} \frac{(2 n+1) !}{p !(2 n+1-p) !} \\
& \times\left(\frac{E_{2}}{E_{3}}\right)^{p} y^{(2 n-1-\beta-2 p) / \beta}
\end{aligned}
$$

where $G_{n}=\frac{(2 n) !}{2^{2 n}(n !)^{2}(2 n+1)}$. Thus, the conditional expectation of $Y$ is

$$
\begin{aligned}
E\left[Y \mid \mathrm{L}_{\text {center }}\right]= & \int_{\alpha[(\sqrt{3}+\gamma) R]^{-\beta}}^{\alpha[\gamma) R]^{-\beta}} y f_{Y}\left(y \mid \mathrm{L}_{\text {center }}\right) d I_{i}=\frac{\alpha M_{0}(\beta, \gamma)}{\gamma^{2} R^{\beta}}
\end{aligned}
$$

where $M_{0}(\beta, \gamma)$ is shown as

$$
M_{0}(\beta, \gamma)=A_{0}(\beta, \gamma)-\frac{2}{\pi} \sum_{n=0}^{\infty} B_{0}(n) \sum_{p=0}^{2 n+1} C_{0}(n, p, \beta, \gamma)
$$

and $A_{0}(\beta, \gamma), B_{0}(n), C_{0}(n, p, \beta, \gamma)$ are

$$
A_{0}(\beta, \gamma)=\frac{\left[(\sqrt{3}-\gamma)^{2-\beta}-(\sqrt{3}+\gamma)^{2-\beta}\right]}{\beta-2}
$$

$$
\begin{gathered}
B_{0}(n)=\frac{(2 n) !}{(2 n+1) 2^{2 n}(n !)^{2}(2 \sqrt{3})^{2 n+1}} \\
C_{0}(n, p, \beta, \gamma)=\frac{\left[(\sqrt{3}+\gamma)^{2 p-2 n-\beta+1}-(\sqrt{3}-\gamma)^{2 p-2 n-\beta+1}\right]}{(2 p-2 n-\beta+1)} \\
\end{gathered}
$$

Similarly, the conditional $m$ th moment of $Y$ can be calculated as

$E\left[Y^{m} \mid \mathrm{L}_{\text {center }}\right]=\int y^{m} f_{Y}\left(y \mid \mathrm{L}_{\text {center }}\right) d y=\frac{\alpha^{m} M_{0}(m \beta, \gamma)}{\gamma^{2} R^{m \beta}}$

For the lognormal shadowing $\mathrm{X}$, the expectation is

$$
E[X]=\int_{0}^{\infty} x \frac{1}{\sqrt{2 \pi} \sigma \lambda x} \exp \left[-\frac{(\ln x)^{2}}{2 \lambda^{2} \sigma^{2}}\right] d x=e^{\frac{\lambda^{2} \sigma^{2}}{2}}
$$

And the $m$ th moment of $X$ is

$$
\begin{aligned}
E\left[X^{m}\right] & =\int_{0}^{\infty} x^{m} \frac{1}{\sqrt{2 \pi} \sigma \lambda x} \exp \left[-\frac{(\ln x)^{2}}{2 \lambda^{2} \sigma^{2}}\right] d x \\
& =\frac{1}{\sqrt{2 \pi} \sigma \lambda} \int_{-\infty}^{+\infty} \exp \left[-\frac{t^{2}}{2 \lambda^{2} \sigma^{2}}+m t\right] d t \\
& =\frac{1}{\sqrt{2 \pi} \sigma \lambda} \int_{-\infty}^{+\infty} \exp \left[-\frac{\left(t-m \lambda^{2} \sigma^{2}\right)^{2}}{2 \lambda^{2} \sigma^{2}}+\frac{m^{2} \lambda^{2} \sigma^{2}}{2}\right] d t \\
& =e^{\frac{m^{2} \lambda^{2} \sigma^{2}}{2}}
\end{aligned}
$$

For the Rayleigh fading $H$, the $m$ th moment is

$$
E\left[H^{m}\right]=\int_{0}^{\infty} h^{m} \mu \mathrm{e}^{-\mu h} d h=\frac{m !}{\mu^{m}}
$$

Hence, it is easy to derive the expectation of $I_{i}$ as

$$
\begin{aligned}
E\left[I_{i} \mid \mathrm{L}_{\text {center }}\right] & =P_{\mathrm{t}, 0} E[X] E\left[Y \mid \mathrm{L}_{\text {center }}\right] E[H] \\
& =\frac{P_{\mathrm{t}, 0} \alpha M_{0}(\beta, \gamma)}{\mu \gamma^{2} R^{\beta}} e^{\frac{\lambda^{2} \sigma^{2}}{2}}
\end{aligned}
$$

With (12), (19), (21), and (22), the conditional $m$ th moment of $I_{i}$ is

$$
\begin{aligned}
E\left[\left(I_{i}\right)^{m} \mid \mathrm{L}_{\text {center }}\right] & =\left(P_{\mathrm{t}, 0}\right)^{m} E\left[X^{m}\right] E\left[Y^{m} \mid \mathrm{L}_{\text {center }}\right] E\left[H^{m}\right] \\
& =\frac{\left(P_{\mathrm{t}, 0} \alpha\right)^{m} M_{0}(m \beta, \gamma) m !}{\mu^{m} \gamma^{2} R^{m} \beta} e^{\frac{m^{2} \lambda^{2} \sigma^{2}}{2}}
\end{aligned}
$$


Then, the conditional variance of $I_{i}$ is given by

$$
\begin{aligned}
V\left[I_{i} \mid \mathrm{L}_{\text {center }}\right]= & E\left[\left(I_{i}\right)^{2} \mid \mathrm{L}_{\text {center }}\right]-\left\{E\left[I_{i} \mid \mathrm{L}_{\text {center }}\right]\right\}^{2} \\
= & \frac{\left(P_{\mathrm{t}, 0} \alpha\right)^{2} e^{\lambda^{2} \sigma^{2}}}{\mu^{2} \gamma^{2} R^{2 \beta}}\left\{2 e^{\lambda^{2} \sigma^{2}} M_{0}(2 \beta, \gamma)\right. \\
& \left.-\frac{\left[M_{0}(\beta, \gamma)\right]^{2}}{\gamma^{2}}\right\}
\end{aligned}
$$

As the interference in sub-band F2 from neighboring cells are i.i.d. RVs, the expectation and variance of $I_{F 2}$ are shown as:

$$
\begin{aligned}
E\left[I_{\mathrm{F} 2}\right]= & \sum_{i=1}^{N} E\left[I_{i} \mid \mathrm{L}_{\text {center }}\right]=\frac{N P_{\mathrm{t}, 0} \alpha M_{0}(\beta, \gamma)}{\mu \gamma^{2} R^{\beta}} e^{\frac{\lambda^{2} \sigma^{2}}{2}} \\
\operatorname{Var}\left[I_{\mathrm{F} 2}\right]= & \sum_{i=1}^{N} \operatorname{Var}\left[I_{i} \mid \mathrm{L}_{\text {center }}\right]=\frac{N\left(P_{\mathrm{t}, 0} \alpha\right)^{2} e^{\lambda^{2} \sigma^{2}}}{\mu^{2} \gamma^{2} R^{2 \beta}} \\
& \times\left\{2 M_{0}(2 \beta, \gamma) e^{\lambda^{2} \sigma^{2}}-\frac{\left[M_{0}(\beta, \gamma)\right]^{2}}{\gamma^{2}}\right\}
\end{aligned}
$$

Considering that $\alpha, \beta$, and $\mu$ are constants, the two statistics of $I_{\mathrm{F} 2}$ are affected by $R, P_{\mathrm{t}, 0}, \gamma$, and $\sigma$. Intuitively, the expectation of $I_{\mathrm{F} 2}$ is proportional to $P_{\mathrm{t}, 0}, R^{-\beta}$, and $e^{\frac{\lambda^{2} \sigma^{2}}{2}}$, while the variance of $I_{\mathrm{F} 2}$ is proportional to $P_{\mathrm{t}, 0}^{2}$ and $R^{-2 \beta}$. The influence of $\gamma$ and $\sigma$ on the expectation and variance of $I_{\mathrm{F} 2}$ will be investigated through numerical results in section 5 .

\section{Statistical analysis of the uplink $\mathrm{ICl}$ in sub-band F1/F3}

In this case, the interfering cells need to be differentiated into two classes, due to different distributing scenarios of interfering sources. In order to describe the two classes, here we choose the ICI in sub-band F1 as an example. From Figure 1, in the cells served by $\mathrm{eNB}_{2}, \mathrm{eNB}_{4}$, and $\mathrm{eNB}_{6}$, the sub-band $\mathrm{F} 1$ will be allocated to only the interior UEs. Differently, $\mathrm{eNB}_{1}, \mathrm{eNB}_{3}$, and $\mathrm{eNB}_{5}$ can allocate sub-band F1 to all the connected UEs. For convenience, we define the cluster of interfering cells which can allocate the given sub-band to only the interior UEs as Set-A, and the cluster of interfering cells which can allocate the given sub-band to all the connected UEs as Set-B. Similarly, in sub-band F3, the neighboring cells will be divided into the two classes also. In Figure 1, the Set-A and Set-B for both sub-band F1 and F3 are presented.

\subsection{PDF of the uplink ICI in sub-band F1/F3}

For both the transmitting power and the path loss depend on the location of interfering UEs, the distribution of interference from the cells of Set-A and the cells of Set-B should be analyzed separately.
For the scenario that interfering UEs are in the cells of Set-A, the corresponding PDF of $I_{i}$ is $f_{I_{i}^{(\mathrm{Set}-\mathrm{A})}}(I)=$ $f_{I_{i}}\left(I \mid \mathrm{L}_{\text {center }}\right)$. In the cells of Set-B, the interfering UEs locate in either the cell center region or the cell edge region. Define the product of $P_{\mathrm{t}}$ and $Y=\alpha D_{i}^{-\beta}$ as $K=$ $P_{\mathrm{t}} Y$. The distribution function of $K$ can be expressed as

$$
P_{\text {Set-B }}(k<K)=P\left(\mathrm{~L}_{\text {center }}, k<K\right)+P\left(\mathrm{~L}_{\text {edge }}, k<K\right)
$$

As we know, the joint distribution functions shown in (28) can be calculated by

$$
\begin{aligned}
& P\left(\mathrm{~L}_{\text {center }}, k<K\right)=P\left(\mathrm{~L}_{\text {center }}\right) P\left(k<K \mid \mathrm{L}_{\text {center }}\right) \\
& P\left(\mathrm{~L}_{\text {edge }}, k<K\right)=P\left(\mathrm{~L}_{\text {edge }}\right) P\left(k<K \mid \mathrm{L}_{\text {edge }}\right)
\end{aligned}
$$

Apparently, the probability of interfering UE being located in the cell center region is $P\left(\mathrm{~L}_{\text {center }}\right)=\gamma^{2}$, and the probability of it being located in the cell edge region is $P\left(\mathrm{~L}_{\text {edge }}\right)=1-\gamma^{2}$. Hence, the PDF of $K$ can be derived from the first derivative of $P_{\text {Set-B }}(k<K)$ as

$$
\begin{aligned}
f_{K^{(\text {Set-B })}}(K) & =\frac{d P_{\text {Set-B }}(k<K)}{d K} \\
& =\gamma^{2} f_{K}\left(K \mid \mathrm{L}_{\text {center }}\right)+\left(1-\gamma^{2}\right) f_{K}\left(K \mid \mathrm{L}_{\text {edge }}\right) \\
& =\frac{\gamma^{2}}{P_{\mathrm{t}, 0}} f_{Y}\left(K / P_{\mathrm{t}, 0} \mid \mathrm{L}_{\text {center }}\right) \\
& +\frac{\left(1-\gamma^{2}\right)}{P_{\mathrm{t}, 1}} f_{Y}\left(K / P_{\mathrm{t}, 1} \mid \mathrm{L}_{\text {edge }}\right)
\end{aligned}
$$

From Figure 2, if the event $\mathrm{L}_{\text {edge }}$ occurs, the conditional CDF of $D_{i}$ equals the ratio between the area of $\Delta_{2}$ and the area of cell edge region. That is

$$
F_{d}\left(D_{i} \mid \mathrm{L}_{\text {edge }}\right)=\frac{S_{\Delta_{2}}}{\pi\left(1-\gamma^{2}\right) R^{2}}
$$

where $S_{\Delta_{2}}$ represents the area of $\Delta_{2}$ in Figure 2 and is given by

$$
S_{\Delta_{2}}= \begin{cases}S_{1} & ,(\sqrt{3}-1) R \leq D_{i}<(\sqrt{3}-\gamma) R \\ S_{1}-S_{\Delta_{1}} & ,(\sqrt{3}-\gamma) R \leq D_{i} \leq(\sqrt{3}+\gamma) R \\ S_{1}-\pi \gamma^{2} R^{2}, & (\sqrt{3}+\gamma) R<D_{i} \leq(\sqrt{3}+1) R\end{cases}
$$

$S_{1}$ represents the overlapping area between the circular region $x^{2}+y^{2} \leq D_{i}^{2}$ and the interfering cell region $(x-$ $\sqrt{3} R)^{2}+y^{2} \leq R^{2}$. It is shown as

$$
\begin{aligned}
S_{1} & =D_{i}^{2} \arccos \left(\frac{D_{i}^{2}+2 R^{2}}{2 \sqrt{3} D_{i} R}\right)+R^{2} \arccos \left(\frac{4 R^{2}-D_{i}^{2}}{2 \sqrt{3} R^{2}}\right) \\
& -\frac{\sqrt{8 R^{2} D_{i}^{2}-D_{i}^{4}-4 R^{4}}}{2}
\end{aligned}
$$




$$
f_{d}\left(D_{i} \mid \mathrm{L}_{\text {edge }}\right)= \begin{cases}\frac{2 D_{i}}{\pi R^{2}\left(1-\gamma^{2}\right)} \arccos \left(\frac{D_{i}^{2}+2 R^{2}}{2 \sqrt{3} R D_{i}}\right) & , D_{i} \in \Phi_{1} \\ \frac{2 D_{i}}{\pi R^{2}\left(1-\gamma^{2}\right)}\left[\arccos \left(\frac{D_{i}^{2}+2 R^{2}}{2 \sqrt{3} R D_{i}}\right)-\arccos \left(\frac{D_{i}^{2}+\left(3-\gamma^{2}\right) R^{2}}{2 \sqrt{3} R D_{i}}\right)\right], D_{i} \in \Phi_{2}\end{cases}
$$

Then, the conditional PDF of $\mathrm{D}_{i}$ when $\mathrm{L}_{\text {edge }}$ occurs can be derived through the first derivative of $F_{d}\left(D_{i} \mid \mathrm{L}_{\text {edge }}\right)$. It is given by

where the two distribution ranges of $D_{i}$ are given by $\Phi_{1}=$ $[(\sqrt{3}-1) R,(\sqrt{3}-\gamma) R) \cup((\sqrt{3}+\gamma) R,(\sqrt{3}+1) R]$, and $\Phi_{2}=[(\sqrt{3}-\gamma) R,(\sqrt{3}+\gamma) R]$.

Then, the conditional PDF of the path loss $Y$ can be derived as

$$
f_{Y}\left(y \mid \mathrm{L}_{\text {edge }}\right)=\frac{(y / \alpha)^{-1 / \beta-1}}{\alpha \beta} f_{d}\left((y / \alpha)^{-1 / \beta} \mid \mathrm{L}_{\text {edge }}\right)
$$

Consequently, we could get that
Similar to (11), the PDF of the total ICI in sub-band F1/F3 shown in (38) can be derived using numerical calculation methods.

\subsection{Expectation and variance of the uplink $\mathrm{ICl}$ in sub-band F1/F3}

Based on the previous analysis, it is known that the expectation and variance of interference from one cell in Set-A are the same as the expressions presented in (23) and (25), respectively. Therefore, the expectation and variance of ICI from a cell in Set-B should be calculated to deduce the two statistics of total ICI in sub-band F1/F3.

Using the same method of power series and polynomial expansion which has been used to derive the substituted expression of $f_{Y}\left(y \mid \mathrm{L}_{\text {center }}\right)$ in subsection 3.2, we could get that

$$
f_{Y}\left(y \mid \mathrm{L}_{\text {edge }}\right)=F_{1} y^{-2 / \beta-1}\left\{\begin{array}{l}
\arccos \left(F_{2} y^{-1 / \beta}+F_{3} y^{1 / \beta}\right), \varphi \in \Phi_{1} \\
\arccos \left(F_{2} y^{-1 / \beta}+F_{3} y^{1 / \beta}\right)-\arccos \left(F_{2} y^{-1 / \beta}+F_{4} y^{1 / \beta}\right), \varphi \in \Phi_{2}
\end{array}\right.
$$

where $\varphi=\left(y / \alpha P_{\mathrm{t}, 1}\right)^{-1 / \beta}, \quad F_{1}=\frac{2(\alpha)^{2 / \beta}}{\pi R^{2} \beta\left(1-\gamma^{2}\right)}$, $F_{2}=\frac{(\alpha)^{1 / \beta}}{2 \sqrt{3} R}, \quad F_{3}=\frac{R}{\sqrt{3}(\alpha)^{1 / \beta}}, \quad F_{4}=\frac{\left(3-\gamma^{2}\right) R}{2 \sqrt{3}(\alpha)^{1 / \beta}}$. Substituting (13) and (36) into (30), the PDF $f_{K^{\text {Set-B }}}(k)$ can be obtained. Then, for the scenario that interfering UEs are in the cells of Set-B, the PDF of $I_{i}$ could be derived as

$$
\begin{aligned}
f_{I_{i}(\mathrm{Set}-\mathrm{B})}(I)= & \int_{0}^{\infty} \frac{1}{h} f_{H}(h) \int_{0}^{\infty} \frac{1}{x} f_{X}(x) f_{K^{(\mathrm{Set}-\mathrm{B})}}(I / x h) d x d h \\
= & \frac{\gamma^{2}}{P_{\mathrm{t}, 0}} \int_{0}^{\infty} \frac{1}{h} f_{H}(h) \int_{0}^{\infty} \frac{1}{x} f_{X}(x) f_{Y} \\
& \times\left[I /\left(P_{\mathrm{t}, 0} x h\right) \mid \mathrm{L}_{\mathrm{center}}\right] d x d h \\
& +\frac{\left(1-\gamma^{2}\right)}{P_{\mathrm{t}, 1}} \int_{0}^{\infty} \frac{1}{h} f_{H}(h) \int_{0}^{\infty} \frac{1}{x} f_{X}(x) f_{Y} \\
& \times\left[I /\left(P_{\mathrm{t}, 1} x h\right) \mid \mathrm{L}_{\text {edge }}\right] d x d h
\end{aligned}
$$

Suppose that $M$ represents the number of cells in Set-A, and $Q$ represents the number of cells in Set-B. The total interference in sub-band F1/F3 is $I_{\mathrm{F} 1 / \mathrm{F} 3}=\sum_{i=1}^{M} I_{i}^{(\mathrm{Set}-\mathrm{A})}+$ $\sum_{i=1}^{Q} I_{i}^{(\text {Set-B) }}$. For the interference from different cells in the same class (Set-A or Set-B) are i.i.d. RVs, the PDF of $I_{\mathrm{F} 1 / \mathrm{F} 3}$ can be calculated by $(M+Q)$-th order convolution as

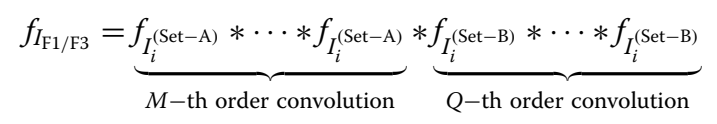

$$
E\left[Y \mid \mathrm{L}_{\text {edge }}\right]=\frac{\alpha M_{1}(\beta)-\alpha M_{0}(\beta, \gamma)}{\left(1-\gamma^{2}\right) R^{\beta}}
$$

where $M_{1}(\beta)$ is shown as

$$
M_{1}(\beta)=A_{1}(\beta)-\frac{2}{\pi} \sum_{n=0}^{\infty} B_{1}(n) \sum_{p=0}^{2 n+1} C_{1}(n, p, \beta)
$$

and $A_{1}(\beta), B_{1}(n), C_{1}(n, p, \beta)$ are given by

$$
\begin{aligned}
& A_{1}(\beta)=\frac{\left[(\sqrt{3}-1)^{2-\beta}-(\sqrt{3}+1)^{2-\beta}\right]}{\beta-2} \\
& B_{1}(n)=\frac{(2 n) !}{(2 n+1) 2^{2 n}(n !)^{2}(\sqrt{3})^{2 n+1}} \\
& C_{1}(n, p, \beta)=\frac{(2 n+1) !}{p !(2 n+1-p) !} * \frac{\left[(\sqrt{3}+1)^{\theta}-(\sqrt{3}-1)^{\theta}\right]}{\theta * 2^{p}} \\
& \theta=2 p-2 n-\beta+1
\end{aligned}
$$

Furthermore, when $L_{\text {edge }}$ occurs, the $m$-th moment of $Y$ is given by

$$
E\left[Y^{m} \mid \mathrm{L}_{\text {edge }}\right]=\frac{\alpha M_{1}(m \beta)-\alpha M_{0}(m \beta, \gamma)}{\left(1-\gamma^{2}\right) R^{m} \beta}
$$


Then, the expectation of interference from a cell in Set-B can be calculated as

$$
\begin{aligned}
E\left[I_{i}^{(\text {Set-B })}\right]= & E\left[K^{(\text {Set-B })}\right] E[X] E[H] \\
= & \left\{P\left(\mathrm{~L}_{\text {center }}\right) P_{\mathrm{t}, 0} E\left[Y \mid \mathrm{L}_{\text {center }}\right]\right. \\
& \left.+P\left(\mathrm{~L}_{\text {edge }}\right) P_{\mathrm{t}, 1} E\left[Y \mid \mathrm{L}_{\text {edge }}\right]\right\} E[X] E[H] \\
= & \frac{P_{\mathrm{t}, 1} \alpha M_{1}(\beta)+\left(P_{\mathrm{t}, 0}-P_{\mathrm{t}, 1}\right) \alpha M_{0}(\beta, \gamma)}{\mu R^{\beta}} e^{\frac{\lambda^{2} \sigma^{2}}{2}}
\end{aligned}
$$

Moreover, the $m$ th moment of interference from one cell of Set-B is

$$
\begin{aligned}
& E\left[\left(I_{i}^{\text {Set-B) }}\right)^{m}\right] \\
& \quad=\frac{\left(P_{\mathrm{t}, 1} \alpha\right)^{m} M_{1}(m \beta)+\left(P_{\mathrm{t}, 0}^{m}-P_{\mathrm{t}, 1}^{m}\right) \alpha^{m} M_{0}(m \beta, \gamma)}{\mu^{m} R^{m \beta}} m ! e^{m^{2} \lambda^{2} \sigma^{2}}
\end{aligned}
$$

With (45) and (46), the variation of interference from one cell in Set-B can be calculated as

$$
\begin{aligned}
V \operatorname{ar}_{\text {Set-B }}\left[I_{i}\right]= & \frac{\alpha^{2} e^{2 \lambda^{2} \sigma^{2}}}{\mu^{2} R^{2 \beta}} 2\left[P_{\mathrm{t}, 1}^{2} M_{1}(2 \beta)\right. \\
& \left.+\left(P_{\mathrm{t}, 0}^{2}-P_{\mathrm{t}, 1}^{2}\right) M_{0}(2 \beta, \gamma)\right] \\
& -\frac{\alpha^{2} e^{\lambda^{2} \sigma^{2}}}{\mu^{2} R^{2 \beta}}\left[P_{\mathrm{t}, 1} M_{1}(\beta)\right. \\
& \left.+\left(P_{\mathrm{t}, 0}-P_{\mathrm{t}, 1}\right) M_{0}(\beta, \gamma)\right]^{2}
\end{aligned}
$$

Using the corresponding statistics of interference from one cell of Set-A and Set-B, the expectation and variance of total interference $I_{\mathrm{F} 1 / \mathrm{F} 3}=\sum_{i=1}^{M} I_{i}^{(\mathrm{Set}-\mathrm{A})}+\sum_{i=1}^{Q} I_{i}^{(\mathrm{Set}-\mathrm{B})}$ are

$$
\begin{aligned}
E\left[I_{\mathrm{F} 1 / \mathrm{F} 3}\right] & =\sum_{i=1}^{M} E_{\text {Set }-\mathrm{A}}\left[I_{i}\right]+\sum_{i=1}^{Q} E_{\text {Set }-\mathrm{B}}\left[I_{i}\right] \\
& =\frac{M P_{\mathrm{t}, 0} \alpha M_{0}(\beta, \gamma)}{\mu \gamma^{2} R^{\beta}} e^{\frac{\lambda^{2} \sigma^{2}}{2}} \\
& +\frac{P_{\mathrm{t}, 1} \alpha M_{1}(\beta)+\left(P_{\mathrm{t}, 0}-P_{\mathrm{t}, 1}\right) \alpha M_{0}(\beta, \gamma)}{\mu R^{\beta}} Q e^{\frac{\lambda^{2} \sigma^{2}}{2}}
\end{aligned}
$$

$$
\begin{aligned}
\operatorname{Var}\left[I_{\mathrm{F} 1 / \mathrm{F} 3}\right]= & \sum_{i=1}^{M} \operatorname{Var}_{\mathrm{Set}-\mathrm{A}}\left[I_{i}\right]+\sum_{i=1}^{Q} \operatorname{Var}_{\mathrm{Set}-\mathrm{B}}\left[I_{i}\right] \\
= & \frac{M\left(P_{\mathrm{t}, 0} \alpha\right)^{2} e^{\lambda^{2} \sigma^{2}}}{\mu^{2} \gamma^{2} R^{2 \beta}}\left\{2 M_{0}(2 \beta, \gamma) e^{\lambda^{2} \sigma^{2}}\right. \\
& \left.-\frac{\left[M_{0}(\beta, \gamma)\right]^{2}}{\gamma^{2}}\right\} \\
& +\frac{2 Q \alpha^{2} e^{2 \lambda^{2} \sigma^{2}}}{\mu^{2} R^{2 \beta}}\left[P_{\mathrm{t}, 1}^{2} M_{1}(2 \beta)\right.
\end{aligned}
$$

$$
\begin{aligned}
& \left.+\left(P_{\mathrm{t}, 0}^{2}-P_{\mathrm{t}, 1}^{2}\right) M_{0}(2 \beta, \gamma)\right] \\
& -\frac{Q \alpha^{2} e^{\lambda^{2} \sigma^{2}}}{\mu^{2} R^{2 \beta}}\left[P_{\mathrm{t}, 1} M_{1}(\beta)\right. \\
& \left.+\left(P_{\mathrm{t}, 0}-P_{\mathrm{t}, 1}\right) M_{0}(\beta, \gamma)\right]^{2}
\end{aligned}
$$

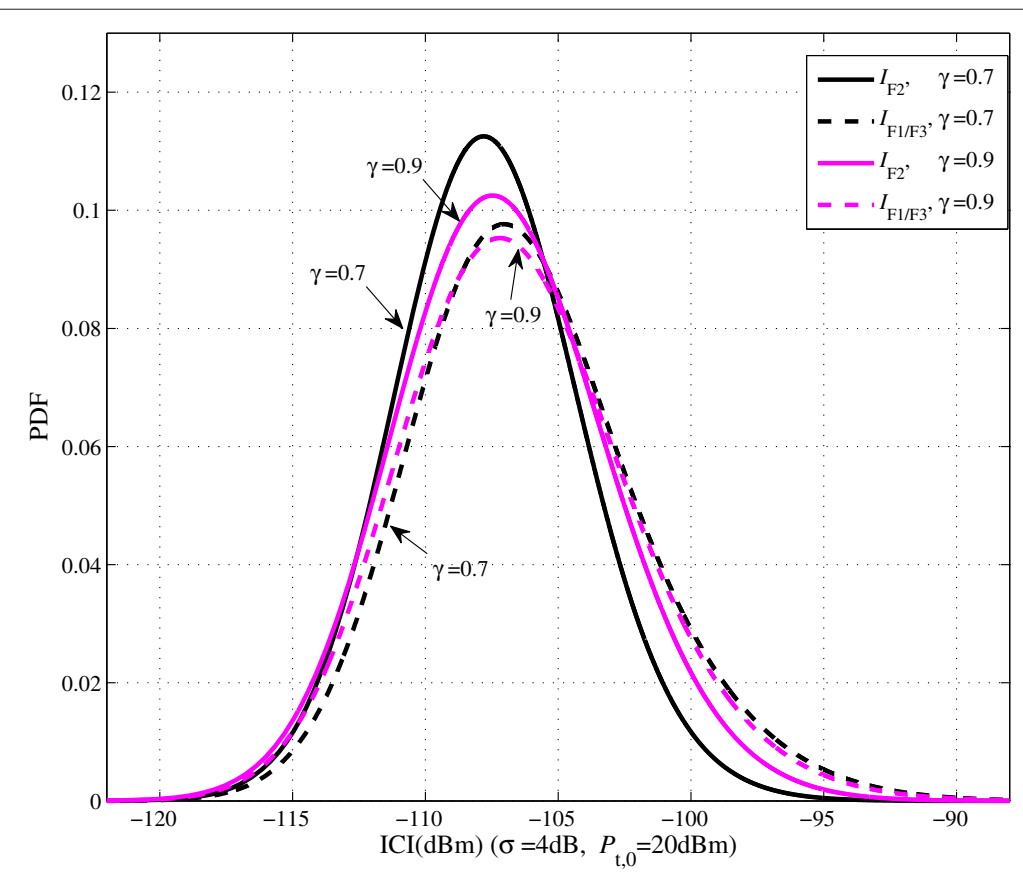

Figure 3 PDF of $\mathrm{ICl}$ with different $\gamma \gamma=(0.7,0.9)$. 
Table 1 System parameters

\begin{tabular}{lc}
\hline Parameter & Assumption \\
\hline Cell radius $R$ & $1,000 \mathrm{~m}$ \\
Transmit power of interior UEs & $P_{\mathrm{t}, 0}=17,20 \mathrm{dBm}$ \\
Transmit power of exterior UEs & $P_{\mathrm{t}, 1}=23 \mathrm{dBm}$ \\
Antenna pattern of UE and eNB & Omnidirectional \\
Antenna gain of UE and eNB & $0 \mathrm{dBi}$ \\
Path loss model & $15.3+37.6 \log _{10} d,(d$ in $\mathrm{m}, \mathrm{dB})$ \\
& $\mathrm{Or} \alpha=0.03, \beta=3.76$ \\
Number of neighboring cells & $N=6$ \\
Number of cells in Set- $\mathrm{A}$ & $M=3$ \\
Number of cells in Set-B & $K=3$ \\
$\gamma$ & $0.7,0.9$ \\
Standard deviation of shadowing & $\sigma=4,6 \mathrm{~dB}$ \\
Rate of Rayleigh fading & $\mu=1$ \\
\hline
\end{tabular}

The derived expressions shown in (48) and (49) are more complicated than the corresponding statistics of $I_{\mathrm{F} 2}$ due to the SFR scheme. Similar to $I_{\mathrm{F} 2}$, the expectation of $I_{\mathrm{F} 1 / \mathrm{F} 3}$ is proportional to $R^{-\beta}$ and $e^{\frac{\lambda^{2} \sigma^{2}}{2}}$, and the variance of $I_{\mathrm{F} 1 / \mathrm{F} 3}$ is proportional to $R^{-2 \beta}$. The impacts of $\gamma, P_{\mathrm{t}, 0}$, and $\sigma$ on the PDF, expectation, and variance of ICI will be investigated through numerical results in the next section.

\section{Analytical and numerical results}

In this section, the impact of system parameters $\left(\gamma, P_{\mathrm{t}, 0}\right.$, and $\sigma$ ) on the PDF and statistics of ICI will be investigated. Furthermore, the theoretical results are compared with Monte Carlo simulations to validate the derivations of statistics.

\subsection{Impact of $\gamma$ on the distribution of ICI.}

As shown in Figure 3, the theoretical PDF of $I_{\mathrm{F} 2}$ and $I_{\mathrm{F} 1 / \mathrm{F} 3}$ with two different values of $\gamma(\gamma=0.7,0.9)$ are presented. The corresponding system parameters are presented in Table 1.

Through the comparison between PDF of $I_{\mathrm{F} 2}$ and $I_{\mathrm{F} 1 / \mathrm{F} 3}$ with same $\gamma$, it is obvious that the distribution of $I_{\mathrm{F} 2}$ concentrates on a relatively low level, while the range of $I_{\mathrm{F} 1 / \mathrm{F} 3}$ is wider in the high interference region. That is because some exterior UEs which are scheduled with sub-band F1/F3 in cells of Set-B may generate severe interference. This difference validates the advantage of SFR scheme on decreasing ICI for exterior UEs. Furthermore, the varying of $\gamma$ has different influence on the distribution of $I_{\mathrm{F} 2}$ and $I_{\mathrm{F} 1 / \mathrm{F} 3}$. Concretely, the distribution of $I_{\mathrm{F} 2}$ becomes more dispersed, and the probability of high interference increases obviously with the increase of $\gamma$. Because the distribution region for the interior UEs is extended, some interfering UEs will be closer to the cell of interest, and some will be further away. However, the distribution of $I_{\mathrm{F} 1 / \mathrm{F} 3}$ tends to move to the low interference region slowly as $\gamma$ increases. For the cells of Set-A, the increase of $\gamma$ has the same effect on the distribution of ICI as $I_{\mathrm{F} 2}$. Whereas, increasing $\gamma$ will reduce the cell edge region for cells of Set-B. Considering that the dominating interfering source of $I_{\mathrm{F} 1 / \mathrm{F} 3}$ are the neighboring exterior UEs, the increase of $\gamma$ will result in lower probability for high interference.

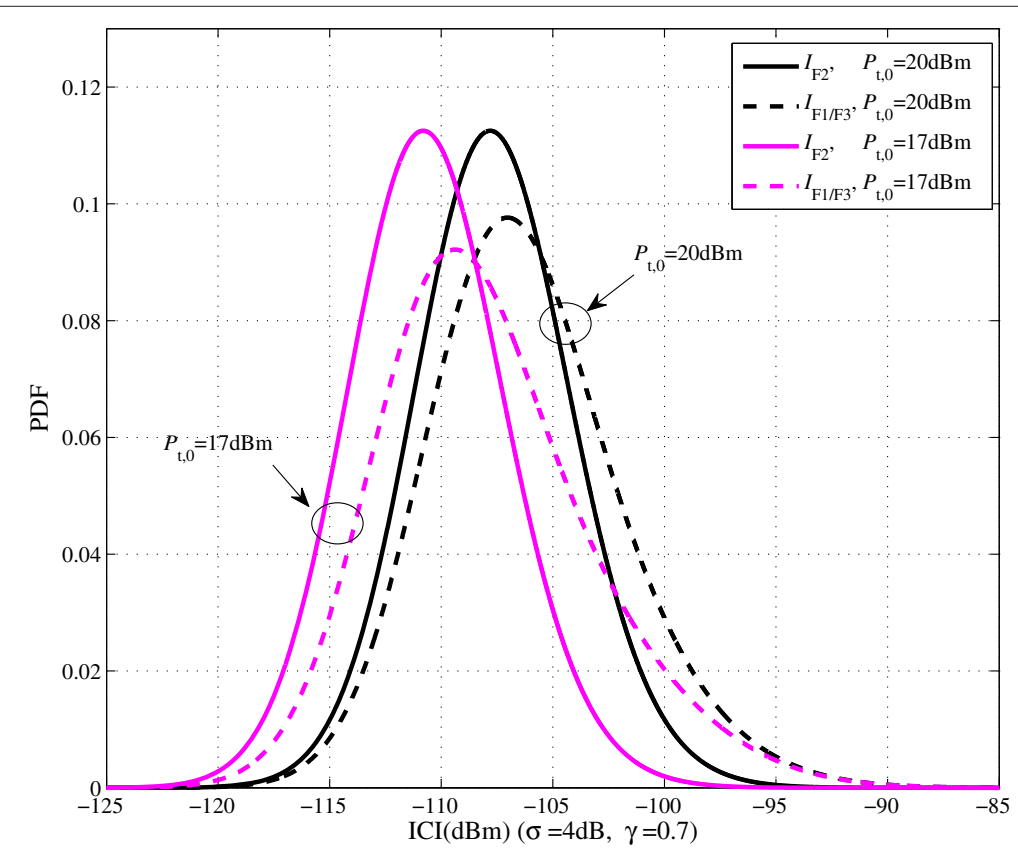

Figure 4 PDF of ICl with different $P_{\mathrm{t}, 0}\left(P_{\mathrm{t}, 0}=17,20 \mathrm{dBm}\right)$. 
Table 2 Settings of $P_{t, 0}, \sigma$, and $\gamma$

\begin{tabular}{lc}
\hline Parameter & \multicolumn{1}{c}{ Assumption } \\
\hline $\begin{array}{l}\text { Transmitting power and standard } \\
\text { deviation of shadowing }\end{array}$ & case 1: $P_{\mathrm{t}, 0}=20 \mathrm{dBm}, \sigma=4 \mathrm{~dB}$ \\
& case 2: $P_{\mathrm{t}, 0}=17 \mathrm{dBm}, \sigma=4 \mathrm{~dB}$ \\
$\gamma$ & case $3: P_{\mathrm{t}, 0}=20 \mathrm{dBm}, \sigma=6 \mathrm{~dB}$ \\
\hline
\end{tabular}

But the tendency of decreasing is not obvious due to the two opposite effects for the different sets of cells (Set-A, Set-B).

\subsection{Impact of $P_{\mathrm{t}, 0}$ on the distribution of $\mathrm{ICI}$}

The PDF curves of $I_{\mathrm{F} 2}$ and $I_{\mathrm{F} 1 / \mathrm{F} 3}$ with different values of $P_{\mathrm{t}, 0}\left(P_{\mathrm{t}, 0}=17,20 \mathrm{dBm}\right)$ are demonstrated in Figure 4. For $I_{\mathrm{F} 2}$ is proportional to $P_{\mathrm{t}, 0}$, the PDF curves of $I_{\mathrm{F} 2}$ moves to the higher interference region without any change of the shape with the increase of $P_{\mathrm{t}, 0}$. Similarly, the PDF of $I_{\mathrm{F} 1 / \mathrm{F} 3}$ near the lower bound moves to the higher interference region with the increase of $P_{\mathrm{t}, 0}$ because the weak interference of $I_{\mathrm{F} 1 / \mathrm{F} 3}$ is mainly determined by the neighboring interior UEs and is proportional to $P_{\mathrm{t}, 0}$. However, the distribution near the upper bound keeps unchanged for the high interference in sub-band F1/F3 is mainly determined by some neighboring exterior UEs. As a result, the distribution of $I_{\mathrm{F} 1 / \mathrm{F} 3}$ becomes more concentrated in the high interference region with the increase of $P_{\mathrm{t}, 0}$.

\subsection{Impact of $\sigma$ on the distribution of ICI}

To investigate the influence of the shadowing on the distribution of ICI, the standard deviation of shadowing is set as $4 \mathrm{~dB}$ or $6 \mathrm{~dB}$ according typical settings of macro model [16]. As depicted in Figure 5, both the distribution of $I_{\mathrm{F} 2}$ and $I_{\mathrm{F} 1 / \mathrm{F} 3}$ are influenced by the shadowing greatly. As the increase of $\sigma$, the distribution of the ICI in both sub-bands becomes more dispersive. The reason is straightforward: large standard deviation of shadowing means the variability of shadowing is great, and it will result in great variability of the received signal or interference.

\subsection{Impact of system parameters on the statistics of ICI} In this part, the statistics of ICI with different system parameters $\left(\gamma, P_{\mathrm{t}, 0}\right.$, and $\left.\sigma\right)$ are compared, and Monte Carlo simulation results are presented to validate the derived expressions of the expectation and variance. Without loss of generality, the standard deviation of ICI is investigated instead of the variance. In addition, it needs to be mentioned that the truncated series of $M_{0}(\beta, \gamma)$, $M_{0}(2 \beta, \gamma), M_{1}(\beta)$, and $M_{1}(2 \beta)$ are calculated with the highest order $n=30$ to get the theoretical calculation results. The settings of $\gamma, P_{\mathrm{t}, 0}$, and $\sigma$ are shown in Table 2 .

The expectation and standard deviation of ICI with different system parameters are depicted in Figure 6 and Figure 7, respectively. The coincidence between theoretical calculations and Monte Carlo simulations validates the derivations on the statistics of ICI. Particularly, $\gamma=1$ means that all cells reuse the whole frequency resources completely. Therefore, the expectation/standard deviation of different sub-bands are same when $\gamma=1$.

From Figure 6, the expectation of $I_{\mathrm{F} 2}$ is smaller than the expectation of $I_{\mathrm{F} 1 / \mathrm{F} 3}$ when $\gamma<1$. Besides that, as $\gamma$

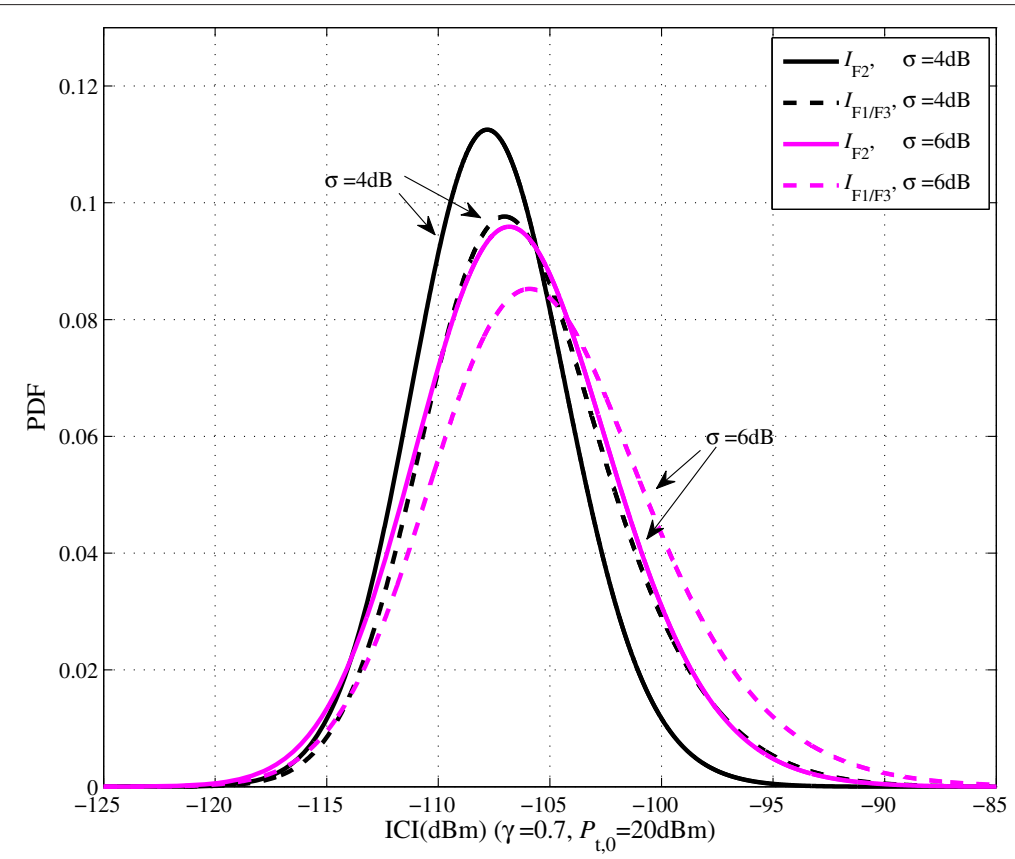

Figure $5 \mathrm{PDF}$ of $\mathrm{ICl}$ with different $\sigma(\sigma=4,6 \mathrm{~dB})$. 


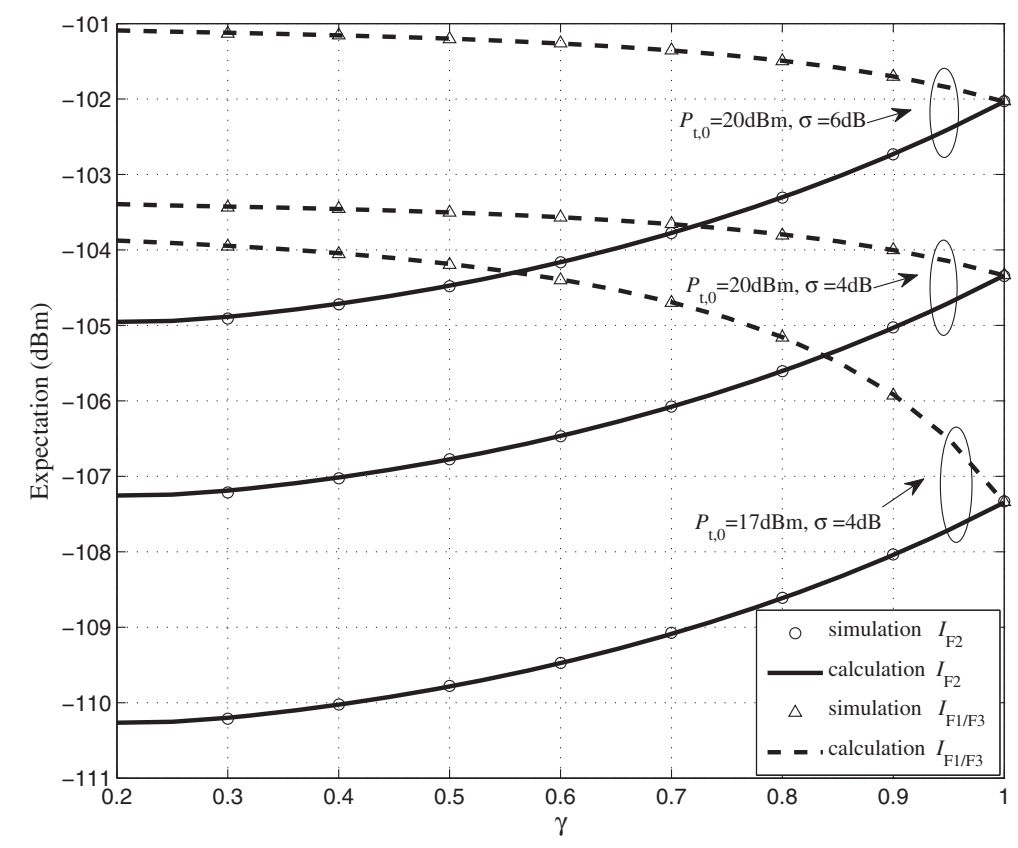

Figure 6 Expectation of $\mathrm{ICl}$.

increases, the expectation of $I_{\mathrm{F} 2}$ tends to increase obviously, but the expectation of $I_{\mathrm{F} 1 / \mathrm{F} 3}$ will decrease slowly. In addition, if $P_{\mathrm{t}, 0}$ is reduced, the expectation of $I_{\mathrm{F} 1 / \mathrm{F} 3}$ will decline faster as $\gamma$ increases. The reason is that the influence of $P_{\mathrm{t}, 0}$ on $I_{\mathrm{F} 1 / \mathrm{F} 3}$ will be more significant if the interior UEs occupy larger quantity in the interfering sources. Differently, the expectation of $I_{\mathrm{F} 2}$ is proportional to $P_{\mathrm{t}, 0}$.
Furthermore, as the shadowing and the path loss are independent, it is observed that larger $\sigma$ will result in the increase of the expectation of $I_{\mathrm{F} 2}$ and $I_{\mathrm{F} 1 / \mathrm{F} 3}$ with the same margin regardless of $\gamma$.

Figure 7 presents the standard deviation curves of $I_{\mathrm{F} 2}$ and $I_{\mathrm{F} 1 / \mathrm{F} 3}$. With the given system parameters, the standard deviation of $I_{\mathrm{F} 2}$ is smaller than the standard deviation

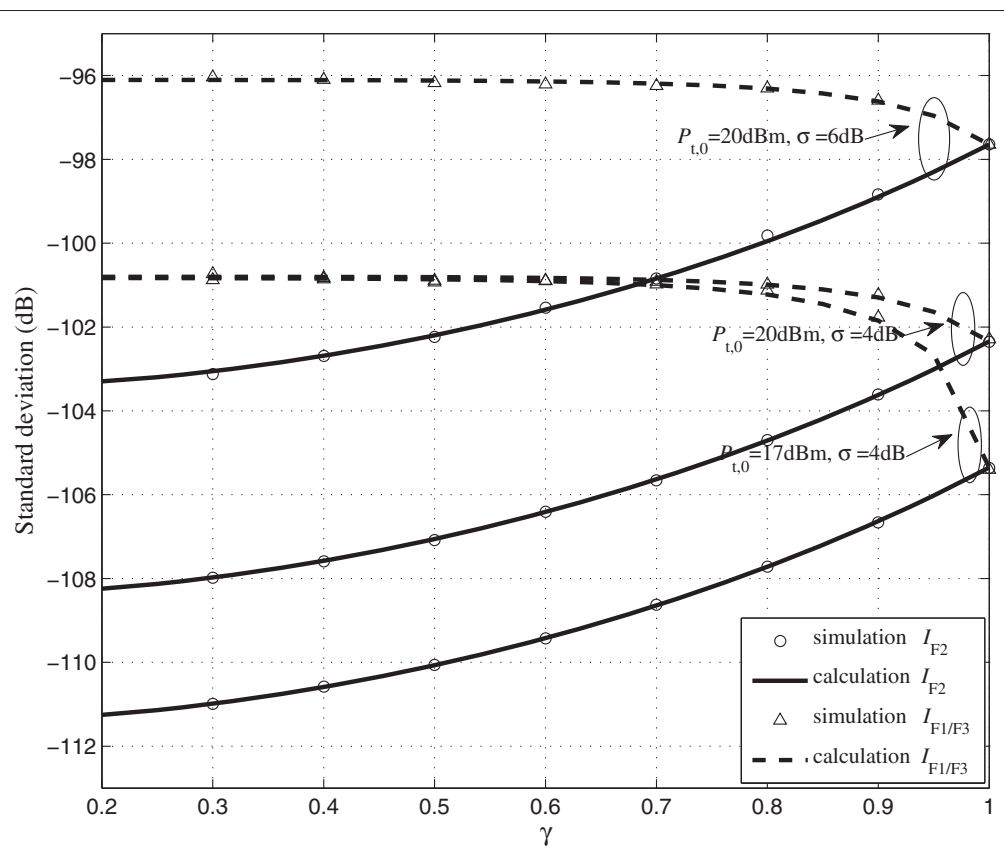

Figure 7 Standard deviation of ICI. 
of $I_{\mathrm{F} 1 / \mathrm{F} 3}$. This demonstrates the higher fluctuation of $I_{\mathrm{F} 1 / \mathrm{F} 3}$, which results from the more flexible scheduling in the cells of Set-B. It needs to be noted that flexible scheduling may result in more diversity gain, but the high fluctuation of ICI will restrict the efficiency of link adaptive technology. Thus, the variance of ICI is important for the system performance evaluation.

Besides that, the standard deviation of $I_{\mathrm{F} 2}$ increases as $\gamma$ for the large cell center region will disperse $I_{\mathrm{F} 2}$ in a wider distribution range. Furthermore, the standard deviation of $I_{\mathrm{F} 2}$ is proportional to $P_{\mathrm{t}, 0}$. However, for $I_{\mathrm{F} 1 / \mathrm{F} 3}$, the standard deviation almost retain the same value if $\gamma<0.8$. Only when $\gamma$ approaches to 1 , the decrease of $P_{\mathrm{t}, 0}$ or the increase of $\gamma$ will result in the decline of the standard deviation of $I_{\mathrm{F} 1 / \mathrm{F} 3}$. Briefly, the effects of $\gamma$ and $P_{\mathrm{t}, 0}$ on the standard deviation of $I_{\mathrm{F} 1 / \mathrm{F} 3}$ become significant only when $\gamma$ is large enough. The reason is that the exterior UEs produce predominant fluctuation of $I_{\mathrm{F} 1 / \mathrm{F} 3}$ generally, but if $\gamma$ approaches to 1 , few scheduling will involve the exterior UEs, then $I_{\mathrm{F} 1 / \mathrm{F} 3}$ will mainly depend on the interior UEs of neighboring cells. In addition, the standard deviation of both $I_{\mathrm{F} 2}$ and $I_{\mathrm{F} 1 / \mathrm{F} 3}$ increase as $\sigma$, and the margin of increase only depends on the shadowing.

\section{Conclusions}

This paper investigates the statistical model of uplink ICI for OFDMA networks with SFR scheme. Through analyzing the distribution of the path loss, shadowing, and Rayleigh fading, the distributions of ICI in different sub-bands are analyzed separately. Then, the closed-form expressions of the expectation and variance of ICI are derived through the method of power series expansion and validated by Monte Carlo simulations. Based on the numerical results, the impacts of system parameters on the PDF and statistics of ICI in different sub-bands are demonstrated. Concretely, for the sub-band scheduled to the exterior UEs in the cell of interest, the varying of $\gamma$ and $P_{\mathrm{t}, 0}$ has a significant effect on the distribution of ICI. For the rest frequency bands, the distribution range of ICI is wider, and the expectation and variance of ICI are much higher; the influence of $\gamma$ and $P_{\mathrm{t}, 0}$ on the expectation of ICI will be significant only when the interior UEs dominate most proportion of interfering sources. Besides that, the standard deviation of shadowing shows a great influence on the distribution of ICI. These derived results can provide guidelines to the design of ICIC scheme parameters and the system performance optimization.

\section{Author details}

${ }^{1}$ Key Laboratory of Wireless Sensor Network \& Communication, Shangha Institute of Microsystem Information and Technology (SIMIT), Chinese Academy of Sciences (CAS), Shanghai 200050, People's Republic of China. ${ }^{2}$ Shanghai Research Center for Wireless Communications (WiCO), Shanghai 200335, People's Republic of China. ${ }^{3}$ University of Chinese Academy of Sciences, Beijing 100049, People's Republic of China.

Received: 6 June 2012 Accepted: 3 April 2013

Published: 1 May 2013

\section{References}

1. Ericsson, in TSG RANWG1 meeting 42. Inter-cell interference handling for E-UTRA, 3GPP R1-050764 (3GPP London, 2005), pp. 1-4

2. F Graziosi, F Santucci, in Proceedings of IEEE Vehicular Technology Conference (VTC). Analysis of second order statistics of the SIR in cellular mobile networks (IEEE Amsterdam, 1999), pp. 1316-1320

3. F Graziosi, L Fuciarelli, F Santucci, in Proceedings of IEEE Vehicular Technology Conference (VTC). Second order statistics of the SIR for cellular mobile networks in the presence of correlated co-channel interferers (IEEE Rhodes, 2001 ), pp. 2499-2503

4. F Graziosi, F Santucci, in Proceedings of IEEE International Conference on Communications (ICC). On SIR fade statistics in rayleigh-lognormal channels (IEEE New York, 2002), pp. 1352-1357

5. FA Ramos, V Ya Kontorovitch, M Lara, in Proceedings of IEEE Vehicular Technology Conference (VTC). On the second order statistics of SIR in wireless Nakagami channels (IEEE Orlando, 2003), pp. 3131-3135

6. KA Hamdi, On the statistics of signal-to-interference plus noise ratio in wireless communications. IEEE Trans. Commun. 57(11), 3199-3204 (2009)

7. C Seol, K Cheun, A statistical inter-cell interference model for downlink cellular OFDMA networks under log-normal shadowing and multipath rayleigh fading. IEEE Trans. Commun. 57(10), 3069-3077 (2009)

8. SN Moiseev, SA Filin, MS Kondakov, AV Garmonov, DH Yim, J Lee, S Chang, YS Park, in Proceedings of IEEE International Conference on Communications (ICC). Analysis of the statistical properties of the SINR in the IEEE 802.16 OFDMA network (IEEE Istanbul, 2006), pp. 5595-5599

9. SN Moiseev, SA Filin, MS Kondakov, AV Garmonov, DH Yim, J Lee, S Chang, YS Park, in Proceedings of IEEE Wireless Communications and Networking Conference (WCNC). Analysis of the statistical properties of the interference in the IEEE 802.16 OFDMA network (IEEE Las Vegas, 2006), pp. 1830-1835

10. KW Sung, H Haas, S McLaughlin, A semianalytical PDF of downlink SINR for femtocell networks. EURASIP J. Wireless Commun. Netw. 2010, 9 (2010)

11. TD Novlan, RK Ganti, A Ghosh, JG Andrews, Analytical evaluation of fractional frequency reuse for OFDMA cellular networks. IEEE Trans. on Wireless Commun. 10(12), 4294-4305 (2011)

12. S Mukherjee, Distribution of downlink SINR in heterogeneous cellular networks. IEEE J. Selected Areas Commun. 30(3), 575-585 (2012)

13. A Musesir, M Bode, KW Sung, H Haas, Analysis SIR for self-organizing wireless networks. EURASIP J. Wireless Commun. Netw. 2009, 8 (2009)

14. I Viering, A Klein, M Ivrlac, M Castaneda, JA Nossek, in Proceedings of IEEE International Conference on Communications (ICC). On uplink intercell interference in a cellular system (IEEE Istanbul, 2006), pp. 2095-2100

15. TD Novlan, HS Dhillon, JG Andrews, Analytical modeling of uplink cellular networks . http://arxiv.org/abs/1203.1304 Accessed 15 Mar 2012

16. 3GPP TR 36.814 v9.0.0. Further advancements for E-UTRA physical layer aspects, (2010)

doi:10.1186/1687-6180-2013-96

Cite this article as: Zhu et al:: Statistical analysis of inter-cell interference in uplink OFDMA networks with soft frequency reuse. EURASIP Journal on Advances in Signal Processing 2013 2013:96.
Competing interests

The authors declare that they have no competing interests.

\section{Acknowledgements}

This work is sponsored by the National Nature Science Foundation of China under grant 61210002 and the International S\&T Cooperation Program of China under grants 2010DFB10410 and 2012DFG11910. 